\title{
SOME INFINITE PERMUTATION GROUPS AND RELATED FINITE LINEAR GROUPS
}

\author{
PETER M. NEUMANN, CHERYL E. PRAEGER, AND SIMON M. SMITH
}

Dedicated to the memory of our late colleague and friend

L. G. (Laci) Kovács

\begin{abstract}
This article began as a study of the structure of infinite permutation groups $G$ in which point stabilisers are finite and all infinite normal subgroups are transitive. That led to two variations. One is the generalisation in which point stabilisers are merely assumed to satisfy min-N, the minimal condition on normal subgroups. The groups $G$ are then of two kinds. Either they have a maximal finite normal subgroup, modulo which they have either one or two minimal non-trivial normal subgroups, or they have a regular normal subgroup $M$ which is a divisible abelian $p$-group of finite rank. In the latter case the point stabilisers are finite and act irreducibly on a $p$-adic vector space associated with $M$. This leads to our second variation, which is a study of the finite linear groups that can arise.
\end{abstract}

\section{INTRODUCTION}

Stimulated by the O'Nan-Scott theory described in [10] of primitive permutation groups that have finite point stabilisers, we initiated a study of infinite permutation groups in which stabilisers are finite and all infinite normal subgroups are transitive. This class includes all primitive, or more generally quasiprimitive, groups with finite point-stabilisers. Although infinite permutation groups with finite stabilisers arise naturally in various contexts they do not usually have the property that their infinite normal subgroups are transitive. A crystallographic group, for example, has finite stabilisers (point groups), but most of its infinite normal subgroups are not transitive on its point-orbits. However, if an infinite permutation group $G$ is primitive (or even if it is no more than quasiprimitive), then a point stabiliser $G_{\alpha}$ is finite if and only if there is a finite upper bound on the lengths of the $G_{\alpha}$-orbitsthis is a special case of a theorem proved by Schlichting [9] and independently by Bergman and Lenstra [1] that gives necessary and sufficient conditions for a transitive group to have a bound on its subdegrees, that is on the lengths of orbits of a point stabiliser.

It was something of a surprise to us that our ideas about groups in which all infinite normal subgroups are transitive and stabilisers are finite could be naturally generalised to those in which the stabilisers merely satisfy min- $N$, the minimal

Date: 10 October 2016.

Key words and phrases. Infinite permutation groups; finite groups; modules; modular representations

MathSciNet Classifications: Primary 20B07; Secondary 20C05, 20C10, 20C20

To appear in J. Aust. Math. Soc. 
condition on normal subgroups. (Philip Hall introduced the notation min- $n$, but $n$ has too many other natural meanings in our mathematics, so we use a variant.)

Throughout this paper $\Omega$ denotes an infinite set, $G$ denotes a subgroup of $\operatorname{Sym}(\Omega)$, and $H:=G_{\alpha}$, the stabiliser of $\alpha$, where $\alpha \in \Omega$. Our focus is on groups satisfying the following conditions:

(C1) all infinite normal subgroups of $G$ are transitive on $\Omega$;

(C2) any non-empty set of normal subgroups of $H$ has a member that is minimal under inclusion, that is, $H$ satisfies min-N.

If all non-trivial normal subgroups of a group $X$ are infinite (equivalently, if $\{1\}$ is the maximal finite normal subgroup of $X$ ) then, for want of a better term, we shall say that $X$ is normally infinite.

Note that any quasiprimitive group of permutations of an infinite set is normally infinite since non-trivial normal subgroups, being transitive, are infinite.

To provide context, here are some simply described, but in some sense representative, examples of groups $G$ satisfying our conditions.

Example 1.1. Let $F$ be an infinite field, let $H:=\mathrm{SL}(2, F)$, and let $V:=F^{2}$ with the natural action of $H$. Take $\Omega:=V$ and $G:=\operatorname{ASL}(2, F)$, the split extension of the translation group of $V$ by $H$. Here $H$ is the stabiliser of 0 and satisfies min- $\mathrm{N}$ (it has centre of order $\leqslant 2$, modulo which it is simple). The translation group is the unique minimal normal subgroup. In this case $G$ is doubly transitive.

Example 1.2. Let $G$ be a simple group acting transitively on an infinite set $\Omega$ such that a stabiliser satisfies min-N (for example, a stabiliser is finite). Or, for a finite group $H$ acting faithfully and transitively on a set $\Gamma$ and an infinite simple group $T$, let $G:=T \mathrm{wr}_{\Gamma} H$ and $\Omega:=T^{\Gamma}$. The action of $G$ on $\Omega$ is the product action of the wreath product, $H$ is a stabiliser, the base group $T^{\Gamma}$ of the wreath product is the unique minimal normal subgroup of $G$ and acts regularly on $\Omega$.

Example 1.3. For any infinite simple group $T$, let $\Omega:=T$, and let $G:=T \times T$ acting by left and right multiplication on $\Omega$ (that is, $\omega^{(a, b)}=a^{-1} \omega b$ ). This has two regular minimal normal subgroups, each isomorphic to $T$, and the stabiliser $H$ of 1 is the diagonal. Then $H \cong T$, so obviously $H$ satisfies min-N.

Example 1.4. For a prime number $p$ let $C_{p^{\infty}}$ denote the Prüfer p-group (isomorphic to $\left.\left\{\theta \in \mathbb{C} \mid \exists k \in \mathbb{N}: \theta^{p^{k}}=1\right\} \leqslant \mathbb{C}^{\times}\right)$. If $\Omega:=G:=C_{p^{\infty}}$ with the regular action, then $G$ has only one infinite normal subgroup, namely $G$ itself, but arbitrarily large finite normal subgroups.

It will be convenient to have some terminology for phenomena illustrated in very basic form by these examples.

- A normally infinite permutation group that has an abelian regular minimal normal subgroup (as in Example 1.1) will be said to be of affine type.

- A normally infinite permutation group that has a unique minimal normal subgroup that is non-abelian (as in Example 1.2) will be said to be of monolithic type.

- A normally infinite permutation group that has precisely two minimal normal subgroups (each of which necessarily acts regularly, as in Example 1.3) will be said to be of bilithic type. 
- If, for some prime number $p$, our group $G$ has a regular normal subgroup that is a divisible abelian $p$-group of finite rank (hence is a direct sum of finitely many copies of $C_{p^{\infty}}$-as in Example 1.4) then $G$ will be said to be of p-divisible affine type.

Before stating our main theorems (to be proved in later sections), we give a further item of contextual information.

Observation 1.1. Suppose that conditions (C1) and (C2) hold. Then G satisfies $\min -\mathrm{N}$.

Proof. Let $\mathcal{N}$ be any non-empty set of normal subgroups of $G$. We show that $\mathcal{N}$ has minimal members. If $\mathcal{N}$ contains any finite normal subgroups of $G$ then it contains one of smallest order, and clearly this is minimal. Suppose now, therefore, that all members of $\mathcal{N}$ are infinite. By the assumption on $G$, they are transitive on $\Omega$. Define $\mathcal{N}_{\alpha}:=\{N \cap H \mid N \in \mathcal{N}\}$. Since $H$ satisfies min-N and all members of $\mathcal{N}_{\alpha}$ are normal subgroups of $H$, there exists $N_{0} \in \mathcal{N}$ such that $N_{0} \cap H$ is minimal in $\mathcal{N}_{\alpha}$. Suppose that $N \in \mathcal{N}$ and $N \leqslant N_{0}$. Then $N \cap H=N_{0} \cap H$ since $N_{0} \cap H$ is minimal in $\mathcal{N}_{\alpha}$ and $N \cap H \leqslant N_{0} \cap H$. Now if $x \in N_{0}$ then since $N$ is transitive on $\Omega$ there exists $y \in N$ such that $\alpha y=\alpha x$, and so $x=\left(x y^{-1}\right) y \in\left(N_{0} \cap H\right)$. $N$, whence (since $\left.N_{0} \cap H=N \cap H\right), x \in N$. Thus $N_{0}=N$ and we have shown that $N_{0}$ is minimal in $\mathcal{N}$. Hence $G$ satisfies min-N.

Note that the Axiom of Choice (AC) is not needed in the above proof. In fact, there are, we believe, only a few places where it is really needed (in some cases in a weak form) in this paper. Those will be noted.

Clearly, in any group $X$, either there are arbitrarily large finite normal subgroups or there is a bound on the sizes of finite normal subgroups. In the latter case, since the product of two finite normal subgroups is a finite normal subgroup there will be a unique maximal (largest) finite normal subgroup $K$ and $X / K$ is normally infinite.

Lemma 1.2. Suppose that conditions $(\mathrm{C} 1)$ and $(\mathrm{C} 2)$ hold, and $G$ has a maximal finite normal subgroup $K$. Then $K$ is semi-regular on $\Omega$ (stabilisers $K_{\omega}$ are trivial for all $\omega \in \Omega$ ). If $\bar{G}:=G / K, \bar{H}:=H K / K \cong H$, and $\bar{\Omega}:=\Omega / K=\Omega / \rho$ where $\rho$ is the $G$-congruence whose blocks are the $K$-orbits, then $\bar{G}$ acts faithfully as a normally infinite group on $\bar{\Omega}$ with stabiliser $\bar{H}$.

This lemma essentially reduces the case where $G$ has a maximal finite normal subgroup to that of normally infinite groups. The following gives a description of normally infinite groups in our context.

Theorem 1.3. Suppose that conditions $(\mathrm{C} 1)$ and $(\mathrm{C} 2)$ hold and $G$ is normally infinite (equivalently, $G$ is quasiprimitive on $\Omega$ ). Then, $G$ has at most two minimal normal subgroups. Moreover, in the language introduced above (p. 2), precisely one of the following holds:

(1) $G$ is of affine type;

(2) $G$ is of monolithic type, and if $M$ is its minimal normal subgroup then $C_{G}(M)=\{1\}$

(3) $G$ is of bilithic type.

In the bilithic case, if $M_{1}, M_{2}$ are the two minimal normal subgroups of $G$, they generate their direct product and if $M_{0}:=H \cap\left(M_{1} \times M_{2}\right)$ then $M_{0}$ is a minimal 
normal subgroup of $H$ and projects isomorphically onto each of $M_{1}$ and $M_{2}$ (hence $M_{1} \cong M_{2}$ and $M_{0}$ is a diagonal of the direct product).

The description given by Lemma 1.2 and Theorem 1.3 of the possibilities in the case that $G$ has a maximal finite normal subgroup probably cannot be developed much further in general.

When $G$ is normally infinite and monolithic $H$ acts faithfully by conjugation as a group of automorphisms of the minimal normal subgroup $M$, and all we know about $M$ is that it must be characteristically simple. One possibility is that it is a direct product of isomorphic simple groups. Then its simple direct factors are minimal normal subgroups of $M$ and they are permuted transitively under the conjugation action of $H$. In this case $G$ looks something like a (perhaps twisted) wreath product of a simple group $T$ by $H$, where $T$ is isomorphic to the simple direct factors of $M$. Indeed, if $M$ acts regularly, that is, if $H \cap M=\{1\}$, then it actually is isomorphic to a (perhaps twisted) wreath product of $T$ by $H$.

Other possibilities are that $M$ could be a variant of the McLain group (see $[3,8]$ ) or one of Philip Hall's wreath powers [5]. A crucial ingredient in the McLain and Hall constructions is an index set that is linearly ordered. In our case the index set needs to be a dense linear ordering whose automorphism group contains a subgroup isomorphic to $H$ having an orbit that is unbounded both above and below. For example, the index set could be $\mathbb{Q}$ with $H=\operatorname{Aut}(\mathbb{Q}, \leqslant)$, a group that certainly satisfies min-N. These are just a few possibilities - it seems probable that there are very many more.

Very similar remarks apply to the bilithic case. Since $M_{0}$ is a minimal normal subgroup of $H$ it is characteristically simple, and any characteristically simple group that can serve as the socle of a monolithic group $G$ could serve as one of the two minimal normal subgroups of a group $G$ of bilithic type. If we strengthen the condition on $H$ and suppose that it satisfies min-SN, the minimal condition on subnormal subgroups (clearly much stronger than min-N), then $M_{0}$ will have minimal normal subgroups. It then follows that $M_{0}$ is a direct product of isomorphic simple groups $T_{i}$ permuted transitively under conjugation by $H$, hence under $H / M$. Thus in this case the minimal normal subgroups $M_{1}, M_{2}$ of $G$ will also be direct products of simple groups, the simple factors in each being permuted transitively under conjugation by $H$ (see Observation 2.1 below). In particular, a little more can be said when $H$ is finite.

Theorem 1.4. Suppose that conditions $(\mathrm{C} 1)$ and $(\mathrm{C} 2)$ hold. If $H$ is finite and $G$ is normally infinite then $G$ is of monolithic type and its monolith $M$ is a direct product $T_{1} \times \cdots \times T_{q}$ of finitely many isomorphic infinite simple groups.

If $G$ does not have a maximal finite normal subgroup then its structure is very different.

Theorem 1.5. Suppose that conditions (C1) and (C2) hold and $G$ has arbitrarily large finite normal subgroups. Then $G$ has a unique minimal infinite normal subgroup $M$, which acts regularly on $\Omega$. For some prime number $p, M$ is a divisible abelian p-group of finite rank (so $G$ is of p-divisible affine type). Moreover, $H$ is finite and acts faithfully and p-adic irreducibly (in the sense of Theorem 4.3 below) by conjugation on $M$. 
REMARK 1. The proof that the rank of $M$ is finite requires AC. When $G$ is of this type, $\Omega$ and $G$ are countably infinite (this also requires AC). Thus if $G$ is uncountable then it must have a maximal finite normal subgroup.

REMARK 2. If $H$ is finite then $G$ is either of $p$-divisible affine type or it is almost monolithic, that is, an extension of a finite normal subgroup $K$ acting semiregularly by a monolithic group $\bar{G}$. The minimal normal subgroup $M$ of $\bar{G}$ will be a direct product of finitely many isomorphic infinite simple groups, permuted transitively under conjugation by $H$. Thus if $M$ acts regularly then $\bar{G}$ will be a wreath product (perhaps twisted) of an infinite simple group by the finite group $H$. This is an immediate consequence of Theorems 1.4 and 1.5.

Proofs of Theorems 1.3 and 1.4 are given in $\S 2$ and a proof of Theorem 1.5 is given in $\S 3$.

Theorem 1.5 leads to an interesting question about finite groups and their modular representation theory. Suppose that $G$ is of $p$-divisible affine type, so that $H$ is finite and $M$ is a divisible abelian $p$-group of finite rank $r$, where $p$ is a prime number. Let $V:=M[p]$, the elementary abelian $p$-group $\left\{x \in M \mid x^{p}=1\right\}$, so that $V$ may naturally be construed as an $\mathbb{F}_{p} H$-module of dimension $r$. A question that naturally arises is: what pairs $(H, V)$ can occur? In other words, what finite (linear) groups can act faithfully (and $p$-adic irreducibly) on divisible abelian $p$-groups of finite rank? We shall show that if $p$ is odd then $H$ must act faithfully on $V$, while if $p=2$ then the kernel of the action of $H$ is an elementary abelian 2-group (Theorem 4.4). Moreover, a given linear group $(H, V)$ can arise from a faithful action on a divisible $p$-group if and only if $V$ is 'liftable' to an integral representation of $H$ (Theorem 4.6).

\section{Proofs of Lemma 1.2 and Theorems 1.3 And 1.4}

Recall the notation and conventions from p. 2. In this section we assume that there is a bound on the sizes of finite normal subgroups of $G$, so that there is a maximal finite normal subgroup $K$ and $G / K$ is normally infinite.

Since $K$ is finite, the group of automorphisms of $K$ induced by the conjugation action of $G$ is finite and therefore $C_{G}(K)$ is a normal subgroup of finite index in $G$, hence infinite and so transitive on $\Omega$. Then since $K$ has a transitive centraliser, $K$ acts semi-regularly. (This is standard-here is the reason. If $x \in K_{\omega}$ and $\omega^{\prime} \in \Omega$ then there exists $y \in C_{G}(K)$ such that $\omega^{\prime}=\omega^{y}$; then $\left(\omega^{\prime}\right)^{x}=\left(\omega^{\prime}\right)^{y^{-1} x y}=\omega^{x y}=$ $\omega^{y}=\omega^{\prime}$, so $x$ fixes every point of $\Omega$, whence $x=1$.)

In the statement of Lemma 1.2 we defined $\rho$ to be the equivalence relation on $\Omega$ whose classes are the $K$-orbits. Since $K \leqslant G, \rho$ is a $G$-congruence and $G$ acts transitively on $\Omega / \rho$. If $L$ is the kernel of this action then $K \leqslant L$. If $L$ were not equal to $K$ then, by the maximality of $K, L$ would be infinite and hence transitive on $\Omega$, which is not the case since $L$ has the same orbits as $K$. Hence the kernel of the $G$-action on $\Omega / \rho$ is $K$. We define $\bar{G}:=G / K, \bar{\Omega}:=\Omega / \rho, \bar{\alpha}:=\alpha K$ and $\bar{H}:=H K / K$. With this notation, $\bar{G}$ acts faithfully on $\bar{\Omega}, \bar{G}_{\bar{\alpha}}=\bar{H}$, all infinite normal subgroups of $\bar{G}$ are transitive, since $\bar{H}$ is a quotient of $H$ (in fact $\bar{H} \cong H$ since $H \cap K=\{1\}$ ), the stabiliser $\bar{H}$ satisfies min-N, and since $K$ is the maximum finite normal subgroup of $G, \bar{G}$ is normally infinite. This completes the proof of the lemma. 
Now assume that $K=\{1\}$, so that $G$ is normally infinite. By Observation 1.1, $G$ satisfies min-N. Suppose first that $G$ has just one minimal normal subgroup $M$. Being infinite $M$ is transitive, so $G=M H$. Since $C_{H}(M)$ is normalised both by $H$ and by $M$ it is normal in $G$, so it is trivial since $H$ contains no non-trivial normal subgroup of $G$. Therefore $C_{H}(M)=\{1\}$, so $C_{G}(M)=M$ or $C_{G}(M)=\{1\}$. If $C_{G}(M)=M$ then $M$ is abelian and $H \cap M \leqslant C_{H}(M)=\{1\}$, and so $M$ acts regularly and $G$ is of affine type. If $C_{G}(M)=\{1\}$ then $M$ is non-abelian and $G$ is of monolithic type. These are cases (1) and (2) of Theorem 1.3.

Now suppose that $G$ has at least two minimal normal subgroups. Let $M_{1}, M_{2}$ be distinct minimal normal subgroups of $G$. Then $M_{1} \cap M_{2}=\{1\}$ so $M_{1}, M_{2}$ centralise each other and generate their direct product. Moreover since each is transitive on $\Omega$, each acts regularly on $\Omega$ and $M_{1}$ is the full centraliser of $M_{2}$ in $\operatorname{Sym}(\Omega)$ (and viceversa - the centraliser in $\operatorname{Sym}(\Omega)$ of a transitive group is transitive if and only if the group acts regularly). Therefore $M_{1}, M_{2}$ are the only minimal normal subgroups and $G$ is of bilithic type. Also, if

$$
M_{0}:=H \cap\left(M_{1} \times M_{2}\right)=\left(M_{1} \times M_{2}\right)_{\alpha}
$$

then $M_{1} \times M_{2}=M_{0} \cdot M_{1}=M_{0} \cdot M_{2}$. Thus $M_{0}$ projects isomorphically onto each of $M_{1}$ and $M_{2}$, and $M_{1} \cong M_{2} \cong M_{0}$.

Let $L$ be a non-trivial normal subgroup of $H$ contained in $M_{0}$ and let $L_{1}, L_{2}$ be the projections of $L$ into $M_{1}, M_{2}$ respectively. Since $L \leqslant M_{0}$ we have $L_{i} \leqslant M_{i}$. Now $H$ also normalises $L_{i}$, and it follows that $L_{i} \preccurlyeq G$. Therefore by minimality of $M_{i}$ we have $L_{i}=M_{i}$ and so $L=M_{0}$. Thus $M_{0}$ is a minimal normal subgroup of $H$.

This completes the proof Theorem 1.3.

Now suppose that $H$ is finite. There exist non-trivial normal subgroups of $M$ whose distinct $H$-conjugates are pairwise disjoint - rather trivially, for example, $M$ itself satisfies this condition. Choose $T \unlhd M$ (and $T \neq\{1\}$ ) such that the distinct $H$-conjugates $T_{1}, T_{2}, \ldots, T_{q}$ of $T$ are pairwise disjoint and furthermore $q$ is as large as possible subject to this condition. By construction, if $i \neq j$ then $T_{i} \cap T_{j}=\{1\}$ so $T_{i}, T_{j}$, being normal subgroups of $M$, commute elementwise. In particular, $T_{1}$ centralises $T_{2} T_{3} \cdots T_{q}$. Let $Z_{1}:=T_{1} \cap\left(T_{2} T_{3} \cdots T_{q}\right)$. Then $Z_{1} \leqslant Z\left(T_{1}\right)$, so $Z_{1}$ is abelian. Now $Z_{1} \leqslant M$ so $Z_{1}$ has only finitely many conjugates $Z_{1}, Z_{2}, \ldots, Z_{s}$ in $G$ and their product $Z_{1} Z_{2} \cdots Z_{s}$ is normal in $G$. Therefore either $Z_{1}=\{1\}$ or $Z_{1} Z_{2} \cdots Z_{s}=M$.

Suppose (seeking a contradiction) that $Z_{1} \neq\{1\}$. Now, being a product of abelian normal subgroups (of itself), by Fitting's Theorem $M$ is nilpotent and its centre $Z$ is a non-trivial abelian normal subgroup of $G$. By minimality $M=Z$, that is, $M$ is abelian. For $a \in M \backslash\{1\}$ the group $\left\langle h^{-1} a h \mid h \in H\right\rangle$ is normalised by both $H$ and $M$, and is therefore a non-trivial normal subgroup of $G$, hence is equal to $M$. Thus $M$ is finitely-generated and abelian. But that is impossible: since there are no non-trivial finite normal subgroups in $G, M$ must be free abelian of finite rank and so $\left\{a^{2} \mid a \in M\right\}$ is a normal subgroup of $G$ properly contained in $M$. This contradiction shows that $Z_{1}=\{1\}$, that is, that $T_{1} \cap\left(T_{2} T_{3} \cdots T_{q}\right)=\{1\}$.

Similarly, of course $T_{i}$ intersects the product of the groups $T_{j}$ for $j \neq i$ trivially. Therefore $M=T_{1} \times T_{2} \times \cdots \times T_{q}$ and $H$ acts by conjugation to permute the factors $T_{i}$ transitively. Let $U_{1}$ be a non-trivial normal subgroup of $T_{1}$ (so $U_{1} \preccurlyeq M$ ). If $h \in H$ then $U_{1}^{h} \leqslant T_{1}^{h}$, so $U_{1}^{h} \leqslant M$ and $\left\langle U_{1}^{h} \mid h \in H\right\rangle \leqslant G$. Since $U_{1}$ has a conjugate inside 
each of $T_{1}, T_{2}, \ldots, T_{q}$, there are at least $q$ conjugates of $U_{1}$ in $M$ which are pairwise disjoint. By the maximality of $q$ therefore, $U_{1}$ has exactly one conjugate $U_{i}$ in $T_{i}$ for each $i \in[1 . . q]$ and, arguing as above, $M=\left\langle U_{1}^{h} \mid h \in H\right\rangle=U_{1} \times U_{2} \times \cdots \times U_{q}$. It follows that $U_{1}=T_{1}$, and so $T_{1}$ is simple. This completes our proof of Theorem 1.4.

A similar argument may be used to show the following.

Observation 2.1. If $G$ is normally infinite of bilithic type and $H$ satisfies min-SN then $M_{0}, M_{1}$ and $M_{2}$ (as in the statement of Theorem 1.3) are direct products of finitely many isomorphic infinite simple groups.

For, normal subgroups of $M_{0}$ are subnormal in $H$, and therefore if $H$ satisfies min-SN then $M_{0}$ satisfies min-N. Let $T$ be a minimal normal subgroup of $M_{0}$. For a finite subset $\Phi$ of $H$ define $P_{\Phi}:=\left\langle T^{h} \mid h \in \Phi\right\rangle$ and $C_{\Phi}:=C_{M_{0}}\left(P_{\Phi}\right)$. Then $P_{\Phi} \leqslant M_{0}$ and so $C_{\Phi} \leqslant M_{0}$. Since $M_{0}$ satisfies min-N, there exists a finite subset $\Psi$ of $H$ such that $C_{\Psi}$ is minimal in the set $\left\{C_{\Phi} \mid \Phi \subseteq_{\text {fin }} H\right\}$. Then $C_{\Psi}=C_{\Psi \cup\{h\}}$ for any $h \in H$, and so $C_{\Psi}=C_{M_{0}}(P)$ where $P:=\left\langle T^{h} \mid h \in H\right\rangle$. Clearly, $P \leqslant H$ and $P \leqslant M_{0}$ and so $P=M_{0}$ since $M_{0}$ is a minimal normal subgroup of $H$. Then $C_{\Psi}=C_{M_{0}}(P)=C_{M_{0}}\left(M_{0}\right)$. If $C_{\Psi}=M_{0}$ then $M_{0}$ is abelian and since it satisfies min-N, it would have to be finite, which is not the case. Therefore $C_{\Psi}=\{1\}$ (being the centraliser of $P$ it is normal in $H$ ). Now if $h \in H$ and $T^{h} \nless P_{\Psi}$ then $T^{h} \cap P_{\Psi}=\{1\}$ (since $T^{h}$ is a minimal normal subgroup of $M_{0}$ ) and so $T^{h}$ would centralise $P_{\Psi}$ which is not the case. Therefore $T^{h} \leqslant P_{\Psi}$ for all $h \in H$, that is, $P_{\Psi}=P$. Thus $M_{0}$ is a product of finitely many conjugates of $T$, and now the proof can be completed as in the case where $H$ is finite.

\section{Proof of Theorem 1.5}

Now suppose that conditions $(\mathrm{C} 1)$ and $(\mathrm{C} 2)$ hold and there are arbitrarily large finite normal subgroups in $G$. Let $\mathcal{K}$ be the set of all finite normal subgroups of $G$ and let $K:=\langle N \mid N \in \mathcal{K}\rangle$. Then $K$ is an infinite normal subgroup of $G$, hence transitive on $\Omega$. Also let $\mathcal{C}:=\left\{C_{G}(N) \mid N \in \mathcal{K}\right\}$. Note that for each $N \in \mathcal{K}$, $C_{G}(N)$ is the kernel of the map from $G$ to $\operatorname{Aut}(N)$ induced by conjugation and so is normal in $G$. Since by Observation $1.1, G$ satisfies min-N, $\mathcal{C}$ contains a minimal member $C$. Then $C=C_{G}(L)$, for some $L \in \mathcal{K}$. Since $L$ is finite, and $G / C \leqslant \operatorname{Aut} L$, $|G: C|$ is finite, so $C$ is infinite, hence transitive. For any $N \in \mathcal{K}$, since $L$ and $N$ are finite normal subgroups of $G$, so also is $N L$ and hence $N L \in \mathcal{K}, C_{G}(N L) \in \mathcal{C}$. Now $C_{G}(N L)$ centralises $L$, that is, $C_{G}(N L) \leqslant C$. Therefore $C_{G}(N L)=C$ by minimality of $C$ in $\mathcal{C}$. It follows that $C$ centralises each $N \in \mathcal{K}$, and hence $C$ centralises $K$. Since both $C$ and $K$ are transitive, we must have $C=C_{G}(K)$, $K=C_{G}(C)$, and both $C$ and $K$ act regularly on $\Omega$.

Let $M:=C \cap K$, the centre $Z(K)$. Since $K \varangle G$ and the centre is a characteristic subgroup, $M \leqslant G$. Since $C$ has finite index in $G, M$ has finite index in $K$, and hence is infinite and therefore transitive. Being a transitive subgroup of the regular groups $C$ and $K$, the group $M$ acts regularly, and it follows that $M=K=C$. Thus $C_{G}(M)=C_{G}(K)=C=M$, so $M$ is abelian and is the unique minimal infinite normal subgroup of $G$. Also, $G=M H$ with $M \cap H=1$, since $M$ is regular, and since $M=C$, it has finite index in $G$. Therefore $H$ is finite and acts faithfully on $M$ by conjugation. 
We next determine the structure of $M$. Since $M$ is abelian we now use additive notation. By the Primary Decomposition Theorem (see, for example, [4, p. 43, Theorem 8.4]) $M=\bigoplus M_{p}$, where the sum is over all prime numbers $p$ and $M_{p}$ is the $p$-primary component of $M$ (recall that $M$, being a union of finite normal subgroups is periodic). Let $p$ be a prime number such that $M_{p} \neq\{0\}$. If $M_{p}$ were finite then $\bigoplus_{q \neq p} M_{q}$ would be infinite. This is a characteristic subgroup of $M$, hence normal in $G$, and therefore transitive. But then we would have $M=\bigoplus_{q \neq p} M_{q}$, which is not the case since $M_{p} \neq\{0\}$. Therefore $M_{p}$ is infinite. As it is a normal subgroup of $G$ it is transitive, so $M_{p}=M$. That is, $M$ is a $p$-group.

For positive integers $n$, define $M[n]:=\{x \in M \mid n x=0\}$. We show next that $M[p]$ is finite. Suppose, seeking a contradiction, that $M[p]$ is infinite. Then it is an infinite normal subgroup of $G$ and so $M[p]=M$, that is, $M$ is elementary abelian of exponent $p$ and infinite rank. We consider $M$ as an $A$-module, where $A$ is the finite-dimensional (indeed, finite) algebra $\mathbb{F}_{p} H$. By Corollary 4.2 below, $M$ contains infinite proper submodules (AC is needed here). These are infinite normal subgroups of $G$ that are not transitive, contradicting our assumption. This proves that $M[p]$ is finite.

It follows that $M$ has finite rank $r$ (equal to the rank of the elementary abelian group $M[p])$. Consider the subgroup $p M$, that is $\{p x \mid x \in M\}$. The map $x \mapsto p x$ is an endomorphism $M \rightarrow M$ and its kernel is $M[p]$, which is finite. Therefore $p M$ is an infinite subgroup of $M$, obviously characteristic, hence normal in $G$. It follows that $p M=M$, that is, that $M$ is $p$-divisible. Being a $p$-group, it is $q$-divisible for all prime numbers $q \neq p$, and therefore it is divisible.

The proof of Theorem 1.5 is now completed by Theorem 4.3 below.

\section{SOME RELEVANT REPRESENTATION THEORY}

We begin with a lemma, and a corollary that is needed in the proof of Theorem 1.5. Recall that the socle $\operatorname{Soc} M$ of a module $M$ is defined to be the submodule generated by all the simple submodules of $M$.

Lemma 4.1. Let $A$ be a finite-dimensional algebra over a field $F$ and let $M$ be an $A$-module. If $\operatorname{dim}(\operatorname{Soc} M)$ is finite then also $\operatorname{dim} M$ is finite.

Proof. Let $J:=\operatorname{Rad} A$, the Jacobson radical defined as the intersection of all the maximal right ideals of $A$. As is well known, $J$ is nilpotent and annihilates any semisimple (right) $A$-module. For an $A$-module $M$ define the ascending Loewy series by

$$
L_{0}:=\{0\}, \quad L_{i+1} / L_{i}=\operatorname{Soc}\left(M / L_{i}\right) \text { for } i \geqslant 0 .
$$

Since $L_{i} J \leqslant L_{i-1}$ for $i \geqslant 1$, it follows easily that $L_{i}=\left\{x \in M \mid x J^{i}=\{0\}\right\}$ for $i \leqslant k$, where $J^{k}=\{0\}$. In particular, $L_{k}=M$. The Loewy length of $M$ is defined to be the smallest $m$ such that $L_{m}=M$.

The assertion of the lemma is trivially true if $M$ is semisimple (Loewy length 1), so suppose as inductive hypothesis that $m>1$ and the assertion is known to be true for modules of Loewy length $\leqslant m-1$. Suppose that the Loewy length of $M$ is $m$ and $\operatorname{dim}(\operatorname{Soc} M)=n$. Let $u_{1}, \ldots, u_{r}$ be generators of $J$. Consider the map $\mu_{i}: L_{2} \rightarrow M, x \mapsto x u_{i}$. Since $u_{i} \in J$ and $L_{2} / L_{1}$ is semisimple, Image $\left(\mu_{i}\right) \leqslant L_{1}$. Therefore $\operatorname{codim}\left(\operatorname{ker} \mu_{i}\right) \leqslant n$. Now

$$
\operatorname{Soc} M=\{x \in M \mid x J=0\}=\operatorname{ker} \mu_{1} \cap \operatorname{ker} \mu_{2} \cap \cdots \cap \operatorname{ker} \mu_{r}
$$


and therefore $\operatorname{codim}_{L_{2}}\left(L_{1}\right) \leqslant r n$. Thus $\operatorname{dim}\left(\operatorname{Soc}\left(M / L_{1}\right)\right)$ is finite. By the inductive hypothesis, $\operatorname{dim}\left(M / L_{1}\right)$ is finite, and therefore $\operatorname{dim} M$ is finite.

Remark. It is clear that one can derive a bound on $\operatorname{dim} M$ in terms of $\operatorname{dim}(\operatorname{Soc} M)$ and $\operatorname{dim} A$ from the above argument. That bound is unrealistically large, however. Using only slightly more sophisticated machinery (see $[2, \S \S 56,57,60])$ we can see that $\operatorname{dim} M \leqslant \operatorname{dim} A \times \operatorname{dim}(\operatorname{Soc} M)$. For, if $\operatorname{Soc} M \cong S_{1} \oplus \cdots \oplus S_{r}$, where the summands $S_{i}$ are simple, then there is an embedding $M \leqslant U_{1} \oplus \cdots \oplus U_{r}$ where $U_{i}$ is the injective hull of $S_{i}$. Now the F-dual $U^{*}$ of an injective A-module $U$ is a projective module over the opposite algebra $A^{\mathrm{op}}$. Since $S_{i}$ is simple, $U_{i}$ is indecomposable, and so $U_{i}^{*}$ is also indecomposable and therefore isomorphic to a summand of the free $A^{\mathrm{op}}$-module of rank 1 . Thus $\operatorname{dim} U_{i}=\operatorname{dim} U_{i}^{*} \leqslant \operatorname{dim} M^{\mathrm{op}}=$ $\operatorname{dim} M$. Therefore $\operatorname{dim} M \leqslant r \cdot \operatorname{dim} M \leqslant \operatorname{dim}(\operatorname{Soc} M) \cdot \operatorname{dim} M$.

Corollary 4.2. If $A$ is a finite-dimensional algebra over a field $F$, and $M$ is an infinite-dimensional $A$-module then $M$ has $2^{\aleph_{0}}$ distinct infinite-dimensional proper submodules.

Proof. By Lemma 4.1, since $A$ is finite-dimensional and $M$ is infinite-dimensional also Soc $M$ is infinite-dimensional. Being a sum of simple submodules, Soc $M$ is actually a direct sum of infinitely many simple $A$-submodules (AC is essential here). Therefore $\operatorname{Soc} M$ contains a direct sum $\bigoplus_{i \in \mathbb{N}} S_{i}$ of simple $A$-modules (the fact that an infinite set contains a countably infinite subset also requires AC, albeit only a weak version). Thus if $I$ is any infinite proper subset of $\mathbb{N}$ then $\bigoplus_{i \in I} S_{i}$ is an infinite-dimensional proper submodule, and different choices of $I$ give different submodules. Since there are $2^{\aleph_{0}}$ different possibilities for $I$ there are $2^{\aleph_{0}}$ different proper infinite-dimensional submodules of Soc $M$, hence of $M$.

Next we turn to the analysis of pairs $(H, M)$ where $M$ is the divisible abelian $p$-group of rank $r$ that is the minimal transitive normal subgroup of $G$ when $G$ is of $p$-divisible affine type, and $H$, a stabiliser in $G$, is finite and acts faithfully on $M$ by conjugation. Since any infinite $H$-invariant subgroup of $M$ is normal in $G$, hence transitive, there are no infinite proper $H$-invariant subgroups of $M$. By the structure theorem for divisible abelian groups (see for example [4, Theorem 23.1] $\mathrm{AC}$ is required for this), $M \cong C_{p^{\infty}} \oplus \cdots \oplus C_{p^{\infty}}$, with $r$ summands, where $C_{p^{\infty}}$ denotes the Prüfer $p$-group (see Example 1.4). It is not hard to see that such a direct sum decomposition of $M$ leads to an isomorphism of the endomorphism ring End $M$ with the algebra $\mathrm{M}\left(r, \hat{\mathbb{Z}}_{p}\right)$ of $r \times r$ matrices over the ring of $p$-adic integers. We may identify $H$ with a subgroup of Aut $M$, and since Aut $M \cong \operatorname{GL}\left(r, \hat{\mathbb{Z}}_{p}\right)$, we have an embedding $H \leqslant \mathrm{GL}\left(r, \hat{\mathbb{Q}}_{p}\right)$, where $\hat{\mathbb{Q}}_{p}$ is the field of $p$-adic rational numbers.

Theorem 4.3. As subgroup of $\mathrm{GL}\left(r, \hat{\mathbb{Q}}_{p}\right), H$ is irreducible.

Note. Under these circumstances we say that $H$ acts $p$-adic irreducibly on $M$, or that $H$ is a $p$-adic irreducible group of automorphisms of $M$. This is the definition that completes the statement of Theorem 1.5.

Proof of the theorem. Consider the Pontryagin dual $M^{*}$ of $M$ defined by $M^{*}:=$ $\operatorname{Hom}_{\mathbb{Z}}\left(M, S^{1}\right)$, where $S^{1}:=\{z \in \mathbb{C}|| z \mid=1\}$. Since $M=r \cdot C_{p^{\infty}}, M^{*}=r \cdot \operatorname{Hom}\left(C_{p^{\infty}}, C_{p^{\infty}}\right)=$ 
$r . \hat{\mathbb{Z}}_{p}$, a free $\hat{\mathbb{Z}}_{p}$-module of rank $r$. Let $W:=\hat{\mathbb{Q}}_{p} \otimes_{\hat{\mathbb{Z}}_{p}} M^{*}$, an $r$-dimensional vector space over $\hat{\mathbb{Q}}_{p}$. Then $M^{*} \leqslant W$ and for every $w \in W$ there exists $k \geqslant 0$ such that $p^{k} w \in M^{*}$, and so we may think of $W$ as $p^{-\infty} M^{*}$. Also,

$$
H \leqslant \operatorname{Aut} M=\operatorname{GL}\left(r, \hat{\mathbb{Z}}_{p}\right)=\operatorname{Aut} M^{*} \leqslant \operatorname{Aut} W=\operatorname{GL}\left(r, \hat{\mathbb{Q}}_{p}\right) .
$$

Let $U$ be a non-zero $H$-invariant subspace of $W$ and let $s:=\operatorname{dim} U$. We aim to prove that $U=W$, that is, $s=r$. To this end define $U_{0}:=M^{*} \cap U$ and

$$
M_{0}:=U_{0}{ }^{\perp}:=\left\{x \in M \mid u(x)=0 \text { for all } u \in U_{0}\right\} .
$$

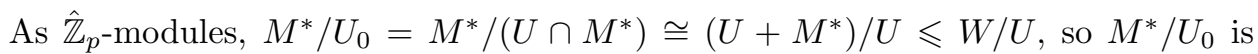
torsion-free. It is also finitely generated. Therefore $M^{*} / U_{0}$ is free since $\hat{\mathbb{Z}}_{p}$ is a principal ideal domain, and so $M^{*} \cong U_{0} \oplus\left(M^{*} / U_{0}\right)$. Thus $U_{0}$ is a free summand of $M^{*}$; it is of rank $s$ since if $u_{1}, \ldots, u_{s}$ is a basis for $U$ then there exists $k \in \mathbb{N}$ such that $p^{k} u_{i} \in M^{*}$ for each $i$ and these $s$ elements are $\hat{\mathbb{Z}}_{p}$-independent. Clearly it is $H$-invariant. It follows easily that $M_{0}$ is an $H$-invariant summand of $M$ of rank $r-s$. There are no infinite proper $H$-invariant subgroups of $M$ and so, since $s \geqslant 1$, it follows that $s=r$ and $U=W$. Thus $H$ is an irreducible subgroup of $\operatorname{GL}\left(r, \hat{\mathbb{Q}}_{p}\right)$, as required.

We turn now to the pair $(H, V)$, where $V=M[p]$ construed as an $\mathbb{F}_{p} H$-module of dimension $r$. Earlier we had erroneously persuaded ourselves that $V$ must be irreducible as $\mathbb{F}_{p} H$-module. That need not be true, as is shown by the following example that we owe to Peter Kropholler and Karin Erdmann.

Example 4.1. The group $H$ generated by the matrix $\left(\begin{array}{rr}0 & 1 \\ -1 & 0\end{array}\right)$ over $\hat{\mathbb{Z}}_{2}$ is cyclic of order 4 and irreducible over $\hat{\mathbb{Q}}_{2}$, and so the split extension of $C_{2 \infty} \oplus C_{2^{\infty}}$ by $H$ has an action of 2-divisible affine type. In this case the action of $H$ on $V$ has kernel of order 2 and $H$ acts reducibly on $V$ as a cyclic group of order 2 .

That $H$ need not act faithfully on $V$ is shown already by the simpler example of the generalised dihedral group $G:=D_{2}$, the split extension of the Prüfer 2-group by a cyclic group of order 2 whose generator acts as inversion. Our next example shows that the kernel $K$ of the action of $H$ on $V$ can be arbitrarily large.

Example 4.2. Let $L:=C_{2 \infty} \oplus C_{2 \infty}$ with the action of $C_{4}$ described in Example 4.1. Let $r:=2 s$ where $s \geqslant 2$, let $M:=s L$ (the direct sum of $s$ groups, each isomorphic to $L)$, and let $H:=C_{4}$ wr $\operatorname{Sym}(s)$. The natural imprimitive action of the wreath product $H$ on $M$ is faithful and 2-adic irreducible. The kernel of the $H$-action on $M[2]$ is $K$, where $K:=C_{2}^{s} \leqslant C_{4}^{s}$. Thus in this example $K$ is an elementary abelian 2-group of order $2^{s}$.

Theorem 4.4. Let $M:=r . C_{p^{\infty}}$, let $V:=M[p]$, and let $H$ be a finite subgroup of Aut $M$. If $p>2$ then $H$ acts faithfully on $V$. If $p=2$ then the kernel $K$ of the action of $H$ on $V$ is an elementary abelian 2-group.

Proof. Let $a \in K$. Consider $a-1 \in \operatorname{End}(M)$. Since $a-1$ annihilates $M[p]$ and the annihilator of $M[p]$ in the endomorphism ring $\operatorname{End}(M)$ is $p \operatorname{End}(M)$, we may write $a=1+p X$ for some $X \in \operatorname{End}(M)$. Suppose now that $a \neq 1$ and (without loss of generality) that $a$ has prime order $q$. Then $X \neq 0$ and so there is a non-negative 
integer $v$ such that $X \in p^{v} \operatorname{End}(M), X \notin p^{v+1} \operatorname{End}(M)$, that is, $v$ is the $p$-adic valuation $v_{p}(X)$ of $X$.

If $q \neq p$ then $1=(1+p X)^{q} \equiv 1+q p X\left(\bmod p^{2 v+2} \operatorname{End}(M)\right)$, whence $q p X \in$ $p^{2 v+2} \operatorname{End}(M)$, which is not the case. Hence $q=p$, and it follows that $K$ is a $p$-group. Next suppose that $p$ is odd. Then

$$
\begin{aligned}
1=(1+p X)^{p} & =1+p^{2} X+\left(\begin{array}{l}
p \\
2
\end{array}\right) p^{2} X^{2}+\cdots+p^{p} X^{p} \\
& \equiv 1+p^{2} X\left(\bmod p^{2 v+3} \operatorname{End}(M)\right) .
\end{aligned}
$$

This implies that $p^{2} X \in p^{2 v+3} \operatorname{End}(M)$, which is false since $v_{p}\left(p^{2} X\right)=v+2$. Therefore $K=\{1\}$ if $p$ is odd.

Suppose now that $p=2$, and that $a \in K$ has order 4 . Then $a=1+2 X$ where $X \neq 0$. Since $a^{2}=(1+2 X)^{2}=1+4 X+4 X^{2} \neq 1$ it follows that if $Y:=X+X^{2}$ then $Y \neq 0$. Let $w:=v_{2}(Y)$. Now $a^{2}=1+4 Y \neq 1$ and

$$
1=a^{4}=(1+4 Y)^{2}=1+8 Y+16 Y^{2} .
$$

Thus $8 Y+16 Y^{2}=0$. Since $v_{2}(8 Y)=w+3$ while $v_{2}\left(16 Y^{2}\right)=2 w+4$, however, this is impossible. Thus $K$ is of exponent dividing 2 and is an elementary abelian 2 -group, as in the statement of the theorem.

Now begin with a prime number $p$ and a pair $(H, V)$, where $H$ is a finite group and $V$ is an $\mathbb{F}_{p} H$-module of dimension $r$. If there exists a divisible abelian $p$-group $A$ of rank $r$ and an embedding $H \leqslant$ Aut $A$ such that $A[p] \cong V$ as $\mathbb{F}_{p} H$-module then we call $A$ a divisible hull of $V$ and write $A=p^{-\infty} V$. In this language the question to be addressed is:

what conditions on the pair $(H, V)$ ensure the existence of a divisible hull $p^{-\infty} V$ ?

By Theorem 4.4 it is necessary that $H$ acts faithfully on $V$ if $p$ is odd and that if $p=2$ then the kernel of the action is an elementary abelian 2-group. This condition is very far from sufficient, however, as Example 4.3 below shows.

Let $M$ be an $R H$-module that is $R$-free of rank $r$ and which is such that $M / p M \cong_{H} V$, where $R$ is some integral domain of characteristic 0 such that $R / p R \cong \mathbb{F}_{p}$. We call $M$ an integral cover of $V$ (in the literature it is also called an $R$-form, but since we do not wish to specify $R$, we prefer a less specific term). The following lemma will prove useful.

Lemma 4.5. If $V$ has an integral cover then also $V^{*}$, the dual $\mathbb{F}_{p} H$-module, has an integral cover.

For, if $M$ is an integral cover of $V$ and $M^{*}:=\operatorname{Hom}_{R}(M, R)$, where $R$ is the relevant integral domain, then $M^{*}$ is also a free $R$-module, and of the same rank $r$. The natural map $R \rightarrow R / p R=\mathbb{F}_{p}$ induces a homomorphism $M^{*} \rightarrow \operatorname{Hom}_{R}\left(M, \mathbb{F}_{p}\right)$ with kernel $p M^{*}$. Every member of $\operatorname{Hom}_{R}\left(M, \mathbb{F}_{p}\right)$ has $p M$ in its kernel, and so there is a natural isomorphism $\operatorname{Hom}_{R}\left(M, \mathbb{F}_{p}\right) \cong \operatorname{Hom}_{\mathbb{F}_{p}}\left(M / p M, \mathbb{F}_{p}\right) \cong \operatorname{Hom}_{\mathbb{F}_{p}}\left(V, \mathbb{F}_{p}\right)=V^{*}$. That is, reduction modulo $p$ provides an isomorphism $M^{*} / p M^{*} \rightarrow V^{*}$. Therefore $V^{*}$ has $M^{*}$ as an integral cover.

In general $V$ need not have either a divisible hull or an integral cover. The two go together, however: 
Theorem 4.6. The finite-dimensional $\mathbb{F}_{p} H$-module $V$ has a divisible hull if and only if it has an integral cover.

Proof. Suppose first that $V$ has an integral cover $M$, an $R H$-module for some integral domain $R$ of characteristic 0 with $R / p R \cong \mathbb{F}_{p}$. Let $F$ be the field of fractions of $R$ and let

$$
S:=p^{-\infty} R:=\left\{a / p^{k} \mid a \in R, k \in \mathbb{N}\right\} \subseteq F .
$$

Then $S$ is a subring of $F$ and $R \leqslant S$. Define $p^{-\infty} M:=S \otimes_{R} M$. Since $M$ is a free $R$-module of rank $r, p^{-\infty} M$ is an $S M$-module that is free of rank $r$ as $S$-module. It contains $M$ as an $R H$-submodule, and $p^{-\infty} M / p M$ is an $R H$-module $A$ with the property that $A[p] \cong M / p M \cong V$ as $\mathbb{F}_{p} H$-module. Thus $V$ has a divisible hull.

Now suppose conversely that $V$ has a divisible hull $A$. Consider the dual group $A^{*}:=\operatorname{Hom}\left(A, C_{p^{\infty}}\right)$. As in the proof of Theorem $4.3, A^{*}$ is an $R H$-module where $R=\hat{\mathbb{Z}}_{p}$ and $A^{*}$ is $R$-free of rank $r$. Each element $\varphi \in A^{*}$ induces a homomorphism $A[p] \rightarrow C_{p^{\infty}}[p]$ and so there is a restriction map $\rho: A^{*} \rightarrow \operatorname{Hom}\left(A[p], C_{p}\right.$ ) (where $C_{p}$ denotes the cyclic group of order $\left.p\right)$. It is not hard to see that $\operatorname{ker} \rho=\{\varphi: A \rightarrow$ $\left.C_{p^{\infty}} \mid A[p] \leqslant \operatorname{ker} \varphi\right\}=p A^{*}$. Therefore $A^{*} / p A^{*} \cong \operatorname{Image}(\rho)=\operatorname{Hom}\left(A[p], C_{p}\right)=V^{*}$. Thus $A^{*}$ is an integral cover of $V^{*}$. Since $V^{* *}=V$, applying Lemma 4.5 to $V^{*}$ we see that $V$ has an integral cover, as required.

Finite groups $H$ with $\mathbb{F}_{p} H$-modules $V$ that have no integral cover (and therefore no $p$-divisible hull) certainly exist:

Example 4.3. If $p \geqslant 5, H:=\mathrm{GL}(2, p)$ and $V$ is the natural 2-dimensional module $\mathbb{F}_{p}^{2}$ then $V$ has no integral cover. For, if $R$ were an integral domain of characteristic 0 with field of fractions $F$, and $M$ an $R H$-module that is $R$-free of rank 2, then $F \otimes_{R} M$ would be an $F H$-module of dimension 2 with $H$ acting faithfully. But $H$ has a subgroup isomorphic to the metacyclic group AGL $(1, p)$ and it is easy to see that this has no faithful representation of dimension $<p-1$ over any field of characteristic $\neq p$. Therefore $H$ has no faithful representation of degree $<p-1$ over $F$.

Comment 1. Let us say that $V$ has a $\mathbb{Z}_{p^{2}}$-hull $p^{-1} V$ if there exists a $\mathbb{Z}_{p^{2}} H$ -

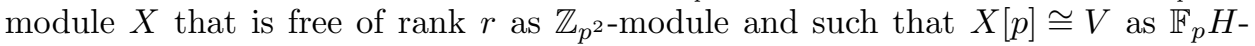
module. The map $x \mapsto p x$ will then be an endomorphism of $X$ with kernel and image both isomorphic to $V$. Define a $\mathbb{Z}_{p^{k}}$-hull analogously. If $Y$ were a $\mathbb{Z}_{p^{3}}$-hull then $p Y$ and $Y\left[p^{2}\right]$ would be 'overlapping' $\mathbb{Z}_{p^{2}}$-hulls. Intuition suggests that if a $\mathbb{Z}_{p^{2-}}$ hull exists then one should be able to manufacture a $\mathbb{Z}_{p^{3}}$-hull from two overlapping copies; then, by some sort of boot-strapping, a $\mathbb{Z}_{p^{k}}$-hull for every $k \geqslant 2$. It should follow that $V$ has a divisible hull $p^{-\infty} V$ if and only if it has a $\mathbb{Z}_{p^{2}}$-hull. Is this true?

Comment 2. Let $H$ be any finite group and $V$ an $\mathbb{F}_{p} H$-module. It is not hard to see from a combination of Theorems 4.3 and 4.6 that the pair $(H, V)$ arises from a permutation group of $p$-divisible affine type if and only if $V$ has an integral cover over some integral domain $R$ of characteristic 0 (not necessarily $\hat{\mathbb{Z}}_{p}$ ) which is rationally irreducible in the sense that it is irreducible as $F H$-module where $F$ is the field of fractions of $R$. Consider the case that $V$ is irreducible. From the beginnings of modular representation theory we see that if $V$ lies in a $p$-block of defect 0 (in the sense that its constituents when $\mathbb{F}_{p}$ is extended to a splitting field lie in blocks of defect 0 ) then $V$ has a rationally irreducible integral cover (or equivalently a 
$p$-adic irreducible integral cover), and therefore $(H, V)$ can arise from a group $G$ of $p$-divisible affine type as in Theorem 1.5. We had hoped that this condition would be necessary as well as sufficient but that is not the case. We are grateful to Karin Erdmann for drawing our attention to examples due to Gordon James (see [6] or [7, Theorem 7.3.23, Example 7.3.26]) of modules of non-zero defect that have integral covers.

Acknowledgements. The first two authors are grateful to the Queen's College, Oxford, All Souls College, Oxford, and the Mathematical Institute, Oxford, for hospitality that facilitated their part of the collaboration. The third author was supported by an Australian Research Council Discovery Early Career Researcher Award (DECRA), project number DE130101521. It is a pleasure to record our gratitude to Karin Erdmann and Peter Kropholler for helpful criticisms of an earlier draft of this article, and also to a conscientious referee for a number of stylistic suggestions.

\section{REFERENCES}

[1] George M. Bergman and Hendrik W. Lenstra Jr, 'Subgroups close to normal subgroups', J. Algebra, 127 (1989), 80-97.

[2] Charles W. Curtis and Irving Reiner, Representation theory of finite groups and associative algebras. John Wiley \& Sons, New York, 1962

[3] Manfred Droste and Rüdiger Göbel, 'McLain groups over arbitrary rings and orderings', Math. Proc. Cambridge Philos. Soc., 117 (1995), 439-467.

[4] László Fuchs, Infinite abelian groups, Vol I. Academic Press, New York and London, 1970.

[5] P. Hall, 'Wreath powers and characteristically simple groups', Proc. Cambridge Philos. Soc., 58 (1962), 170-184 (also in Collected Works of Philip Hall, K. W. Gruenberg and J. E. Roseblade eds, Clarendon Press, Oxford 1988, pp.611-625).

[6] Gordon James, 'On a conjecture of Carter concerning irreducible Specht modules', Math. Proc. Cambridge Philos. Soc., 83 (1978), 11-17.

[7] Gordon James and Adalbert Kerber, The representation theory of the symmetric group. (Encyclopedia of Mathematics and its Applications, 16), Addison-Wesley Publishing Co., Reading, Mass., 1981.

[8] D. H. McLain, 'A characteristically simple group', Proc. Cambridge Philos. Soc., 50 (1954), 641-642.

[9] G. Schlichting, 'Operationen mit periodischen Stabilisatoren', Arch. Math. (Basel), 34 (1980), 97-99.

[10] Simon M. Smith, 'A classification of primitive permutation groups with finite stabilisers', $J$. Algebra, 432 (2015), 12-21.

Peter M. Neumann, The Queen's College, Oxford OX1 4AW, UK

e-mail: peter.neumann@queens.ox.ac.uk

Cheryl E. Praeger, School of Mathematics and Statistics,

The University of Western Australia, 35 Stirling Highway,

Crawley WA 6009, Australia

e-mail: cheryl.praeger@uwa.edu.au

Simon M. Smith, School of Mathematics and Physics, College of Science, University of Lincoln,

Brayford Pool, Lincoln, LN6 7TS, UK 
e-mail: sismith@lincoln.ac.uk 